\title{
NUEVA DOCUMENTACIÓN CONTEXTUALIZADA SOBRE FERNANDO REMACHA (*1898; †1984)
}

\author{
NEW CONTEXTUALIZED DOCUMENTS ON FERNANDO REMACHA \\ $(* 1898 ; \dagger 1984)$
}

\author{
Marcos Andrés Vierge \\ Universidad Pública de Navarra
}

\begin{abstract}
Resumen
El presente artículo aporta nueva documentación sobre Fernando Remacha (1898-1984). En primer lugar, se contrasta la información de dos reseñas críticas de La libertad y El Sol con una copia incompleta de una obra de Remacha depositada en el Archivo Eresbil de Errentería. En segundo lugar, se transcriben, analizan y contextualizan 9 cartas escritas en 1959. Finalmente, se transcribe y contextualiza una carta del compositor Agustín González Acilu dirigida a Remacha en 1967.

\section{Palabras clave}

Fernando Remacha, Agustín González Acilu, Federico Sopeña, Semanas Gregorianas de Pamplona, Orquesta Santa Cecilia.
\end{abstract}

\section{Introducción}

El presente artículo aporta nueva documentación sobre Fernando Remacha que es contextualizada históricamente e interpretada y contrastada con otra documentación ya referencial. A la luz de esta nueva información se refuerzan aspectos ya dichos sobre Remacha referidos a su biografía musical y se aportan algunos datos nuevos que son contrastados con nuevas referencias bibliográficas o recientes trabajos de investigación.

En primer lugar, se muestran dos reseñas críticas sobre una obra "perdida", calificativo utilizado debido a que únicamente hemos localizado hasta ahora una copia incompleta

\begin{abstract}
This paper provides new information about Fernando Remacha (1898-1984). First, information is contrasted two critical reviews of La Libertad and $\mathrm{El} \mathrm{Sol}$ with a incomplete copy of a composition Remacha Eresbil deposited in the Archives of Errentería. Second, are transcribed, analyzed and contextualized nine letters written in 1959. Finally, transcribed and contextualizes a letter the composer Agustín González Acilu Remacha directed in 1967.
\end{abstract}

\section{Key Words}

Fernando Remacha, Agustín González Acilu, Federico Sopeña, Gregorian Weeks of Pamplona, Santa Cecilia Orchestra.

de una obra de Remacha localizada en el Archivo Eresbil de Errentería ${ }^{1}$.

En segundo lugar, se presentan 9 cartas fechadas en 1959, procedentes del domicilio de Remacha en Pamplona, pasando posteriormente al domicilio particular de la hija del compositor, Margarita Remacha y entregados finalmente al

1 En 1996 el Archivo Eresbil de Errentería adquirió la parte de piano de una copia de una obra cuyo encabezamiento dice textualmente: Piano/Suite/Remacha. Hasta el momento no se había localizado ninguna otra información sobre esta composición. 
autor de este artículo 2 . Los documentos seleccionados pueden agruparse con un criterio cronológico y temático tal y como se muestra en la siguiente tabla ${ }^{3}$.

\begin{tabular}{|l|l|l|l|}
\hline FECHA Y LUGAR & EMISOR & RECEPTOR & ASUNTO \\
\hline $\begin{array}{l}15 / 01 / 1959 \\
\text { PAMPLONA }\end{array}$ & Fernando Remacha & Antonio Baciero & $\begin{array}{l}\text { Concierto en Orense } \\
\text { de Antonio Baciero. }\end{array}$ \\
\hline $\begin{array}{l}\text { 02/03/1959 } \\
\text { PARÍS }\end{array}$ & Armando Alfonso & Fernando Remacha & $\begin{array}{l}\text { Oferta concierto a través de } \\
\text { Salvador Bacarisse. }\end{array}$ \\
\hline $\begin{array}{l}\text { 21/02/1959 } \\
\text { PAMPLONA }\end{array}$ & Fernando Remacha & $\begin{array}{l}\text { Rvdo. Bergés de } \\
\text { Leyre }\end{array}$ & $\begin{array}{l}\text { Semanas gregorianas } \\
\text { de Pamplona. }\end{array}$ \\
\hline $\begin{array}{l}\text { 17/03/1959 } \\
\text { PAMPLONA }\end{array}$ & Fernando Remacha & Jesús Guridi & Orquesta Santa Cecilia \\
\hline $\begin{array}{l}\text { 25/03/1959 } \\
\text { PAMPLONA }\end{array}$ & Martín Lipúzcoa & Fernando Remacha & $\begin{array}{l}\text { Semanas gregorianas } \\
\text { de Pamplona. }\end{array}$ \\
\hline $\begin{array}{l}\text { 30/04/1959 } \\
\text { PAMPLONA }\end{array}$ & Fernando Remacha & $\begin{array}{l}\text { Rvdo. Bergés de } \\
\text { Leyre }\end{array}$ & Semanas gregorianas \\
\hline $\begin{array}{l}\text { 10/05/1959 } \\
\text { MADRID }\end{array}$ & Federico Sopeña & Fernando Remacha & Propuesta de conferencia. \\
\hline $\begin{array}{l}\text { 15/05/1959 } \\
\text { PAMPLONA }\end{array}$ & Fernando Remacha & Federico Sopeña & $\begin{array}{l}\text { Respuesta a propuesta } \\
\text { de Sopeña. }\end{array}$ \\
\hline $\begin{array}{l}\text { 30/05/1959 } \\
\text { SAN SEBASTIÁN }\end{array}$ & $\begin{array}{l}\text { CCC (Centro de Cultura por } \\
\text { correspondencia) }\end{array}$ & Fernando Remacha & $\begin{array}{l}\text { Curso de Armonía } \\
\text { a distancia. } .\end{array}$ \\
\hline
\end{tabular}

Por último, se transcribe y contextualiza una carta del compositor Agustín González Acilu a Fernando Remacha, fechada en Madrid el 14.06.1967.

\section{Suite para nueve instrumentos y voz, de Fernando Remacha}

En una crítica musical publicada en el diario $\mathrm{La} \mathrm{Li-}$ bertad, Sainz de la Maza menciona una Suite para nueve

2 Todos los documentos fueron entregados al Archivo del Conservatorio Pablo Sarasate de Pamplona y del Conservatorio Superior de Navarra. Ambos centros cuentan en la actualidad con un único archivo. Por otra parte, otros trabajos vinculados a interpretación y contextualización de documentación epistolar de Fernando Remacha son: ANDRÉS, 1998b: 29-92. ANDRÉS, 2010b: 75-85.

3 No obstante, aunque en el apartado de "asunto" se describa exactamente el objetivo de la correspondencia, el interés de cada carta, desde nuestro enfoque de trabajo, puede no ser el mismo.

$4 \mathrm{Al}$ igual que las anteriores esta carta procede del domicilio de Pamplona de Fernando Remacha. instrumentos y voz de Remacha, de la que destaca la alta elaboración y depuración compositiva: "Música de línea y expresión latinas, de elocuencia sin retórica, y sobre todo, bien cimentada, sin vacíos, como quiere su maestro, el gran Malipiero"5.

Por otro lado, el crítico Adolfo Salazar se refería a dicha obra en el diario $\mathrm{El} \mathrm{Sol}$ de la siguiente manera:

"Aunque la Suite de Fernando Remacha parecería obligarle a esa especie de "declaración de principios" que supone la música que hoy se hace evocando modelos dieciochescos, este músico prefiere guardar su libertad y sólo muy de lejos alude a minués, gavotas y passapiés, que apenas hacen sino ofrecerle un motivo de carácter, desarrollado después liberalmente. El segundo trozo es un Aria con voz, cantada por Concepción Badía, un tanto gris e indeciso de hechura".

5 SAINZ DE LA MAZA, 1933: 8.

6 SALAZAR, 1933: 5. 
En 1996, el Archivo de Compositores Vascos Eresbil de Errentería adquirió una partitura cuya portada lleva por titulo: Piano /Suite /Remacha ${ }^{7}$. No se trata de un manuscrito original de Remacha sino de una copia con 17 páginas en formato vertical. La obra está estructurada en las siguientes secciones: "Minuit", "Air", "Gavotti Tacci", "Passipiid". Aunque en algunos fragmentos, como sucede en la primera sección, la partitura parece contener toda la música, dado que en otros pasajes se escriben anotaciones para otros instrumentos (flauta, violín primero, viola y voz), lo más lógico es que se trate de la parte de piano de una obra con más conjunto. Las indicaciones de otros instrumentos sirven para que el pianista pueda saber lo que hay justamente delante de una parte que interpreta (Figura 1).
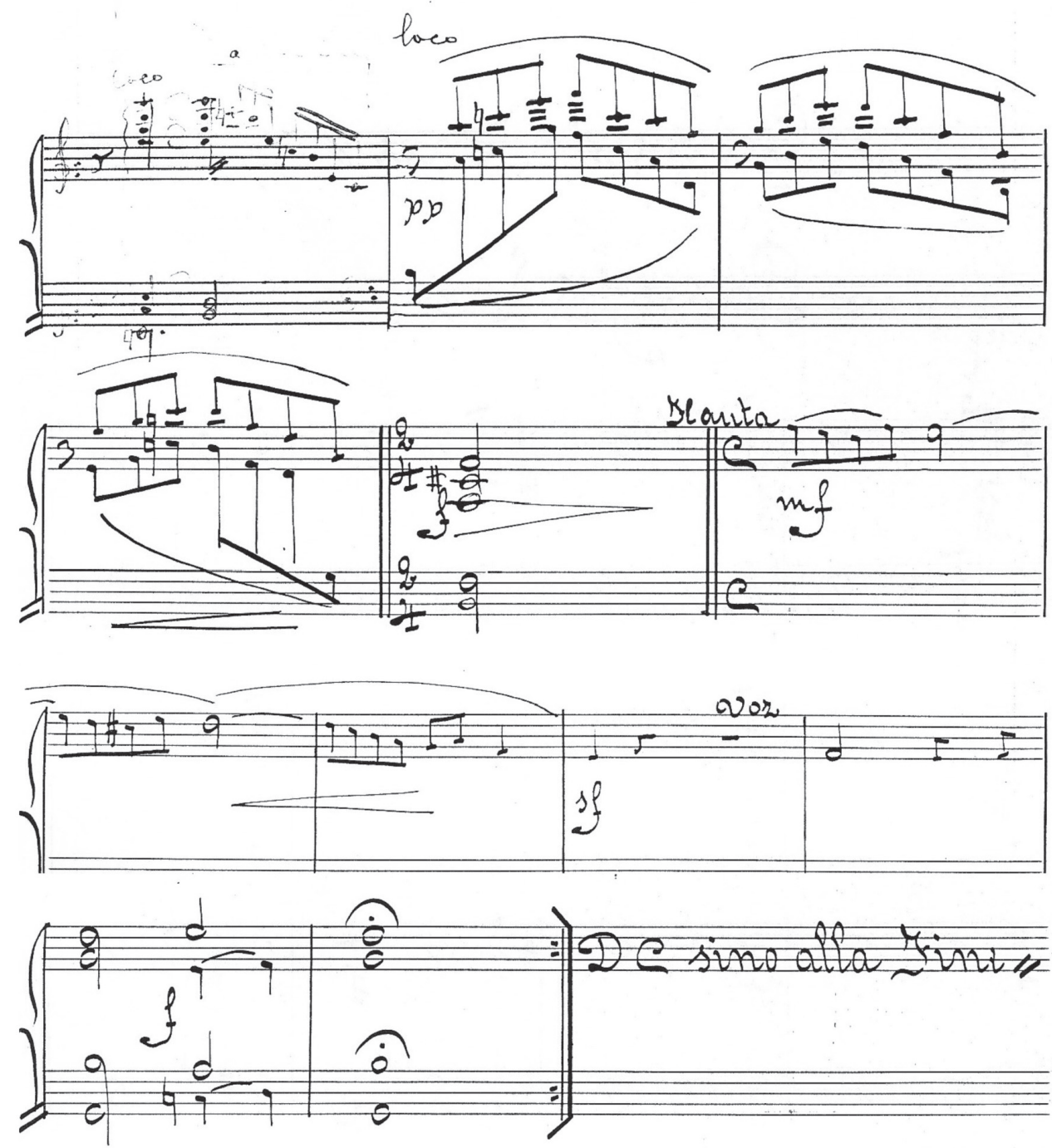

Fig. 1: parte del Aria de la Suite

7 Este dato fue incluido en el catálogo de Remacha de la siguiente tesis doctoral defendida en la Universidad de Valladolid: ANDRÉS, 1997: 592.

8 Aunque el título del "passipiid" está inmediatamente después de la "Gavotti". 
Por tanto, se tenía constancia de una suite de Remacha que por escritura o lenguaje era fácilmente contextualizada en los años posteriores a su estancia en Roma (1928) y anteriores a la guerra civil. De hecho, hay claras semejanzas en aspectos melódicos y armónicos con la Suite para violín y piano $(1928)^{9}$. Como ya explique en otro artículo, Julio Arce fecha el estreno de la Suite para violín y piano el 09.11.1928. $\mathrm{Al}$ no existir partitura manuscrita de esta obra, la referencia que utiliza Arce es una reseña crítica de la revista Ondas ${ }^{10}$. Del mismo modo, las dos reseñas críticas que aporta este artículo permiten, por un lado, verificar que efectivamente la partitura comprada por Eresbil es una copia incompleta de una obra de Remacha ${ }^{11}$ (Sainz de la Maza menciona nueve instrumentos y voz), por otro, contextualizar la pieza en los años citados. Además, la lectura de ambas críticas permite situar la interpretación de la obra en un concierto realizado bajo los auspicios de la Sociedad de Cursos y Conferencias en el nuevo "Auditórium" de la Residencia de Estudiantes, el domingo 11.06.1933, con Gustavo Pittaluga al frente del grupo de cámara y solistas de la Orquesta Filarmónica, con la colaboración de la cantante Concepción Badía y el pianista Enrique Aroca ${ }^{12}$.

Como es sabido, el Archivo Histórico de la Residencia desapareció, lo que imposibilita una reconstrucción más exacta de los hechos y por el momento el hallazgo de la partitura completa. No obstante, la recepción de la obra resulta en este caso concreto fundamental para verificar cierta información y contextualizar algunos datos.

Respecto al contenido de las dos reseñas críticas, aparecen calificaciones y referencias que ya podemos considerar clásicas en la recepción de la obra de Remacha. Así, hay que subrayar la referencia a Malipiero, el laconismo de la escritura de Remacha y muy especialmente lo que dice Salazar en el sentido de que aunque Remacha parta de ideales estéticos concretos (en este caso, una estética neoclásica), siempre se muestra como un compositor muy libre, a lo que se añade aquí, muy original y fantasioso.

\section{Documentación epistolar del año 1959}

La secuencia biográfico-musical de Remacha puede establecerse en dos grandes períodos: previo y posterior a

9 El hecho de no disponer de todas las partes imposibilita un análisis significativo de la obra, aunque se puedan hacer apreciaciones de vocabulario armónico y perfil melódico.

10 ARCE, 2008: 105.

11 Con una grafía diferente a la copia de la Suite para violín y piano.

12 Para contextualizar la importancia para la "Música Nueva" de la Residencia de Estudiantes, véase: PALACIOS, 2008: 114-118. la guerra. Antes de la guerra, hay que diferenciar al menos tres etapas: su estancia en Madrid, realizando estudios musicales y tomando contacto con el resto de compositores que luego se presentarían como el Grupo de Madrid; el período romano, como alumno becado en la Academia Española en Roma, trabajando con su maestro Malipiero, estancia que tiene lugar desde 1923 a 1928; finalmente, los años madrileños anteriores a la guerra, momentos de reconocimiento público (dos premios nacionales) y de asentamiento laboral en la productora Filmófono como compositor de bandas sonoras.

Después de la guerra, se pueden diferenciar al menos dos grandes etapas: la posguerra en Tudela, hasta 1957, trabajando en el negocio familiar (una ferretería) y la etapa de Pamplona, como director del Conservatorio Pablo Sarasate, años que recuperan definitivamente a Remacha para el espacio público, gestionando diversos frentes musicales que aunque muy productivos para la vida musical de Navarra, merman enormemente su labor compositiva.

La documentación epistolar que se presenta a continuación pertenece al año 1959, momento en el que Remacha ha iniciado ya una gestión del conservatorio que no se limita a la "enseñanza reglada", sino que aspira a una verdadera extensión o proyección social del centro musical. Como ejemplo, cabe citar la organización de las Semanas Gregorianas, a las que ya me he referido en otros sitios y sobre las que el reciente trabajo de Milagros Flamarique ofrece una visión evolutiva y social sumamente interesante ${ }^{13}$.

\section{Carta de Fernando Remacha a Antonio Baciero, 15.01.1959}

La presente carta tiene un interés relacionado con la suite para piano Cartel de Fiestas ${ }^{14}$. Como ya expliqué en otro texto, Baciero se refiere a que fue él quien estrenó Cartel de Fiestas para piano, en el Museo de Navarra el 17.11.1959. Sin embargo, una reseña periodística de Diario de Navarra, con fecha 02.05.1953, narra un concierto que se celebró el día anterior en homenaje a Remacha, organizado por la Real Cofradía del Gallico de San Cernin, en el que María Dolores Malumbres interpretó al piano Cartel de Fiestas $^{15}$.

Lo que aporta la presente carta es que Baciero interpretó efectivamente la suite Cartel de Fiestas o probablemente parte de ella ese mismo año de 1959 en Orense, en un

13 FLAMARIQUE, 2011.

14 Por otra parte, la carta se relaciona con otra carta dirigida a Guridi que es comentada más adelante.

15 ANDRÉS, 2009: 128. 
concierto organizado por Antonio Iglesias, lo que reafirma que efectivamente era una obra que tenía en programa, aunque en ningún momento se aluda a su interpretación como obra de estreno. Por otro lado, resultan muy interesantes las palabras del propio Remacha al referirse al segundo movimiento, como excesivamente descriptivo, razón por la cual sugiere a Baciero que elimine esa parte de la obra para su interpretación. Esta apreciación estética por parte del propio compositor resulta curiosa si se tiene en cuenta que es el único movimiento de la obra en la que no se utiliza material popular ni citas claramente descriptivas, como por ejemplo, en Chupinazos ${ }^{16}$.

\section{Carta de Armando Alfonso a Fernando Remacha, 02.03.1959}

Esta carta tiene interés porque contrastada con la documentación que Heine presentó sobre la correspondencia entre Remacha y Salvador Bacarisse en 1949 y 1953, ayuda a comprender el cambio y la nueva posición que adquirió Remacha como director del conservatorio navarro. Las duras palabras que Remacha escribe a Bacarisse sobre su posición social y vital en $1949^{17}$ debían de quedar ya muy lejanas para el compositor navarro que en 1959 había adquirido una condición laboral completamente diferente.

\section{Tres cartas vinculadas a la Primera Semana Gregoriana}

Dos de las tres cartas que se comentan en este apartado están dirigidas al Reverendo Dom. Bergés del Monasterio de Leyre ${ }^{18}$. La tercera de ellas la escribió Martín Lipúzcoa a Remacha. Lipúzcoa, presbítero y director del Orfeón Pamplonés durante los años 1948 a 1956, fue uno de los principales artífices para que Remacha asumiera el cargo de director

16 Una reciente grabación de la versión sinfónica de la obra es la siguiente: TEMES, 2009.

17 HEINE, 1996: 240-241.

18 El Padre Albert Bergés fue un monje benedictino de la Abadía de San Martín de Ligugé (Poitiers-Francia), donde murió y está enterrado. Con Leyre mantuvo siempre una excelente relación. Antes de la restauración de la Vida Benedictina en Leyre, vivió varios años en el monasterio de Cogullada (Zaragoza), filial de su monasterio de Ligugé hasta los años 1932-33 en que fue suprimido. Después de la restauración de la vida monástica en Leyre, en1954, pasó allí largas temporadas. Posteriormente retornó a Francia y cumplió diversos servicios y ministerios como capellán de monjas en Córcega. Terminó sus días en Ligugé, víctima de un cáncer. Por otra parte, tal y como señala Milagros Flamarique, los actos de clausura de cada curso de las Semanas Gregorianas de Pamplona se celebraban con una misa cantada por los cursillistas en estilo gregoriano y los dos primeros años, la solemne misa de clausura se celebró en el Monasterio de Leyre, mientras que el resto, la misa fue oficiada en la Catedral de Pamplona: FLAMARIQUE, 2011: 34. del Conservatorio Pablo Sarasate de Pamplona ${ }^{19}$. Además Lipúzcoa fue Profesor de canto gregoriano y polifonía religiosa en el Conservatorio "Pablo Sarasate" y director de la Schola Cantorum del Seminario de Pamplona.

Desde la perspectiva actual, cabe destacar la visión o ideario del conservatorio que tenía Remacha, razón por la que a su labor docente y directiva hay que añadir otra de gestión cultural del centro musical hacia la sociedad del momento muy importante.

La primera carta, dirigida a Bergés el 21.02.1959 aporta el dato histórico de que en los trámites para contratar para la Semana Gregoriana de aquel año a dos personalidades del momento, Dom Gajard ${ }^{20}$ y H. Potiron ${ }^{21}$, intervino decisivamente precisamente Albert Bergés del Monasterio de Leyre.

La segunda carta dirigida a Bergés el 30.04.1959 interesa por la visión de Remacha de la "Semana" intentando proyectar publicidad para revistas especializadas de música francesas como la histórica Revue Musicale ${ }^{22}$. La contextualización histórica del momento, desde el ostracismo cultural particular de una provincia como Navarra, subyace de alguna manera en las palabras de Remacha cuando dice lo siguiente: "Perdone que le moleste tanto pero no se puede conseguir anunciar en Francia sino por medio de personas que estén dentro del país".

La última carta de este apartado escrita por Martín Lipúzcoa a Remacha el 25.03.1959 complementa las anteriores en el sentido de que se refiere a la publicidad nacional de la "Semana", recomendando publicar el anuncio del evento en el Boletín Oficial Diocesano ${ }^{23}$.

\section{Carta de Fernando Remacha a Jesús Guridi, 17.03.1959}

Esta breve carta de Remacha a Jesús Guridi resulta interesante porque muestra un hecho hasta ahora desco-

19 ANDRÉS, 1998a: 213.

20 Dom Joseph Gajard (1885-1972): monje y musicólogo francés, perteneciente a la orden benedictina, maestro de coro de la abadía de Solesmes y director de La Revue du Chant Grégorienne.

21 Henri Potiron (1882-1972): profesor del Instituto Gregoriano de París. Compositor y gregorianista francés especializado principalmente en acompañamiento organístico y modalidad gregoriana y autor de varios tratados sobre ambas disciplinas.

22 Hay que recordar las palabras de Remacha cuando se refería a que a través de la Revue Musicale le entusiasmó la figura de Malipiero. Véase: ANDRÉS, 2009: 125-126. Consultado el número de junio de 1959 al que hace referencia Remacha no se ha encontrado publicidad del curso de Pamplona.

23 Flamarique dedica un apartado de su trabajo a la difusión de las Semanas Gregorianas en revistas y prensa nacional, regional, así como a los comunicados a sedes religiosas, episcopales y conventuales. FLAMARIQUE, 2011: 53-55. 
nocido, que es la labor del compositor tudelano vinculada a la organización de conciertos de la Orquesta Santa Cecilia.

Tal y como explica Jaime Berrade, la Sociedad de Conciertos Santa Cecilia, pasó por un período de crisis o inestabilidad importante durante los años 1945-198524. En concreto en 1958 ,

"La Junta directiva, presidida por Félix Huarte, conocido empresario y mecenas, buscó el apoyo de instituciones administrativas y sociales, hasta el punto de celebrar una reunión presidida por el Diputado Sr. Asiáin, a la que concurrieron representantes de la Institución Príncipe de Viana y del Patronato del Conservatorio de Música Pablo Sarasate, centro educativo igualmente dependiente de la administración foral. Esta especie de gabinete de crisis orientó una nueva remesa de subvenciones forales y municipales"25.

Lo que se contextualiza con las siguientes palabras de Remacha a Guridi y verifica una vez más que la relación del empresario y mecenas Félix Huarte-Remacha se proyectaba en muchas más acciones que la dirección del conservatorio: "Mi distinguido amigo: por encargo del Patronato que ahora se ha hecho cargo de organizar conciertos con la Orquesta de Santa Cecilia, me dirijo a Vd. Para rogarle que nos preste el archivo del Conservatorio (...)".

Igualmente, esta carta se relaciona con la primera comentada en este apartado, dirigida a Baciero y que refleja una situación previa: "Lo de la Orquesta de aquí sigue sin prisa. Ahora van a pedir ayuda a ciertas Sociedades Industriales para ligar la publicidad a la ayuda que puedan prestar".

\section{Carta de Federico Sopeña a Fernando Remacha, 10.05.1959 y contestación de Remacha a Sopeña, 15.05.1959}

Esta breve carta de Sopeña a Remacha interesa porque refleja el apoyo temprano e indiscutible al centro que dirige Remacha, por parte de una personalidad musical española del momento como es Federico Sopeña. Cabe destacar que estas palabras de aliento cobraron una dimensión mayor, cuando Sopeña escribió un amplio artículo elogiando el Conservatorio Pablo Sarasate coincidiendo con la inauguración del nuevo local en la calle Aoiz en $1963^{26}$.

24 BERRADE, 2011: 150-157.

25 BERRADE, 2011: 152.

26 SOPEÑA, 1963: 20-23.
Respecto a la contestación que hizo Remacha a Sopeña, manifestó la imposibilidad para ese año de llevar a cabo la conferencia de Sopeña sobre Puccini, pero en cambio, le invitó para el próximo año a participar como profesor de la Semana Gregoriana, lo que se hizo realidad en la edición de $1960^{27}$.

\section{Carta del Centro de Cultura por Correspondencia a Fer- nando Remacha, 30.05.1959}

Sorprende esta notificación de CCC por el hecho de rechazar la oferta por parte de Remacha de un curso de armonía, "lamentando no poder aceptarlo por disponer ya de un original preparado para la imprenta". Por el momento, no se tiene constancia de un curso anterior al de Remacha publicado por dicho centro. En cambio, en 1964 la empresa Polyglophone CCC de San Sebastián inició la edición de un Curso de Armonía a distancia impartido por Fernando Remacha que es un ejemplo de síntesis y precisión técnica ${ }^{28}$.

\section{Carta de Agustín González Acilu a Fernando Remacha, 14.06.1967}

La referencia bibliográfica fundamental para el estudio de González Acilu es el trabajo de Marta Cureses $^{29}$. No obstante la carta que se presenta aquí íntegramente por primera vez tiene interés, desde el enfoque de este trabajo, por la perspectiva que con el paso del tiempo se va fraguando en dos biografías vinculadas y separadas al mismo tiempo, la vida y estética de Fernando Remacha, la biografía y obra de González Acilu. Es sabido que Remacha ofreció a Acilu la posibilidad de dirigir el conservatorio de Pamplona, algo a lo que el compositor de Alsasua renunció. Igualmente, es conocida la ayuda que desde su posición de filtro con la Diputación Foral de Navarra que establecía Remacha, ayudó a diferentes artistas como Acilu para su proyección profesional. Por otra parte, no cabe duda de que las propuestas estéticas de Acilu no fueron y difícilmente podían serlo, propias de Remacha y sin embargo este nunca vaciló en

\footnotetext{
27 FLAMARIQUE, 2011: 43.
}

28 REMACHA, 1964. En 1939, de la mano de Víctor Zabala, nace en San Sebastián (España) la "Academia CCC". Durante los años sesenta los cursos de CCC adquieren un perfil más técnico, ampliándose nuevamente en consonancia con la demanda social. La metodología a distancia permitió a los trabajadores más humildes cualificarse para poder acceder a puestos que requerían un título determinado o un conocimiento de idioma. Es en este contexto cuando se inicia el curso de Remacha.

29 CURESES, 2001. 
apoyarlas. González Acilu tuvo la posibilidad de estudiar en Italia, precisamente con una beca de la Diputación Foral de Navarra, lo que le permitió conocer nuevas tendencias que tenían que ver con la experimentación fonética. En este sentido, la carta que aquí se presenta hace referencia al proceso compositivo de Dilatación fonética, obra que supuso un nuevo camino técnico y estético para el compositor del Alsasua (como así lo atestiguan las propias palabras del compositor). A través de este documento se ofrece íntegro un documento que revela la propuesta poética y el procedimiento compositivo de Acilu. Como puede observarse, la preocupación por la percepción musical, tan propia de mucha música de la segunda mitad del siglo XX está presente ya en aquellos años en González Acilu. Otros procedimientos que han preocupado a compositores de esta época, también. Es el caso de la utilización de los cuartos de tono o las duraciones, vinculadas para Acilu, en este caso, a la altura de los sonidos ${ }^{30}$.
Por otra parte, la posible obra para la que Acilu solicita ayuda a Remacha para conseguir una subvención probablemente es la que finalmente se constituyó como encargo del Instituto Alemán de Madrid y que Acilu dedicó a Remacha: Aschermittwoch (1968). Muchos años más tarde, el 14.12.2009 González Acilu participó en un concierto organizado por la Universidad Pública de Navarra en memoria de Fernando Remacha, cuyo objeto principal era inaugurar el Aula Magna de dicha institución precisamente con el nombre del compositor tudelano ${ }^{31}$. Acilu participó en esta ocasión con Límites II para piano, cuya numeración hace referencia a Límites I, composición estrenada el 14.12.2009 en un concierto en memoria de Ramón Barce ${ }^{32}$.

Sin duda alguna la participación de Acilu en este homenaje-concierto abrió las puertas para que al año siguiente el compositor de Alsasua fuera nombrado Doctor Honoris Causa por la Universidad Pública de Navarra, acto que tuvo lugar precisamente en el Aula Magna Fernando Remacha.
30 Puede verse un trabajo reciente vinculado a este asunto en: ANDRÉS, 2010a.
31 ANDRÉS, 2010c.

32 ANDRÉS, 2010c: 21. 


\section{Anexos: transcripciones ${ }^{33}$ escaneos $^{34}$}

\section{Carta de Fernando Remacha a Antonio Baciero}

Querido amigo: recibo tu carta y me apresuro a contestarte para que sepas que Filare no puede acompañarte a Orense ni yo tampoco. Creo que debes de conseguir de Iglesias que no haya conferencia sino notas al programa.

Me parece bien el programa del concierto que has enviado a la Sra. Drewsen y te ruego que en el Cartel de Fiestas suprimas el segundo número por parecerme que es demasiado fácil por ser tan descriptivo. Con tres números hay suficiente.

Lo de la Orquesta de aquí sigue sin prisa. Ahora van a pedir ayuda a ciertas sociedades industriales para ligar la publicidad a la ayuda que puedan prestar los conciertos. Pero aun pensando en echar a andar aún lo veo lejano. Ya hablaré con Troncoso mañana que hay Patronato para ver si se acelera el "debut". Tiene más autoridad que nadie pues Príncipe de Viana les ayuda con 100.000 pesetas. Ya le propondré también lo de los dos conciertos tuyos. Ya te avisaré como cae.

Escríbele a Don Félix dándole cuenta de los planes y fechas. Ayer estuvo tu padre a invitarme a ir el domingo pero me es imposible. Te deseo el mayor éxito y debías de intentar que graben en cinta el concierto para mandárselo a Don Félix y poder oírlo también aquí y hasta radiarlo.

Recibe un fuerte abrazo $^{35}$.

\section{Carta de Armando Alfonso a Fernando Remacha}

París, 2 de Marzo de 1959

Sr. D. Fernando Remacha

Director del Conservatorio "Pablo Sarasate"

Muy distinguido Sr. Mió:

Si bien no le conozco personalmente, sí lo hago a través de sus obras y por las abundantes referencias que me llegan de mis excelentes amigos Salvador y Pilar Bacarisse, los cuales me han insistido mucho para que me dirija a Vd. hablándole de un asunto que me interesa.

Se trata de que en el mes de Abril voy a realizar una gira por España, dirigiendo la Orquesta de Cámara Internacional de París. En esta gira actuaremos en Valladolid, Madrid, Valencia, Gerona y otras ciudades españolas y naturalmente, puesto que necesariamente habremos de pasar no muy lejos, nos gustaría y nos interesaría actuar en Pamplona. Bacarisse opina que Vd. podría hacer algo en ese sentido, este es el motivo por el que me permito molestarle, esperando no ocasionarle grandes trastornos.

La orquesta de que le hablo es una orquesta de cuerda de 16 músicos de siete nacionalidades diferentes, casi todos de menos de 30 años y competentísimos todos ellos, ya que, además de su experiencia profesional, ostentan la mayoría importantes distinciones académicas: primeros premios del Conservatorio de París, licencia de la Ecole Normale, Premios "Sarasate", diplomas de honor de la Escuela Julliard de Nueva York, etc.

Nuestro viaje por España tendrá lugar en la primera quincena de Abril y los momentos en que, con toda seguridad nos vendría bien pasar por Pamplona serían los últimos días de Marzo, inmediatamente después de la Semana Santa, o bien quizá los últimos días de la primera quincena de Abril.

33 Todas las cartas escritas por Remacha presentadas en este trabajo están mecanografiadas, en formato apaisado y en papel de cebolla. Por otra parte, en toda la documentación transcrita se han corregido cuestiones de ortografía y se han suprimido espacios superfluos.

34 No se presenta escaneada toda la documentación sino aquella que se considera especialmente de interés, bien sea por tratarse de documentación manuscrita, bien por ser especialmente relevante su contenido. Por otra parte, en la actualidad los dos conservatorios navarros cuentan con un mismo archivo pero no se ha procedido por el momento a inventariar sus fondos. No obstante la documentación vinculada a la tesis de Marcos Andrés Vierge está agrupada y organizada en carpetas relacionadas con ese trabajo, igualmente que la nueva documentación que se aporta aquí.

35 "Filare" era el seudónimo de Alberto Fraile, crítico musical durante muchos años de El Pensamiento Navarro. Iglesias es Antonio Iglesias, músico y director del Conservatorio de Orense en aquellos momentos. Por otra parte, existe una amplia correspondencia entre Helga Drewsen referida a las Semanas de Música Antigua de Estella [véase ANDRÉS, 1997: Anexos]. Miguel Troncoso era jefe de la Sección de Música de Príncipe de Viana y uno de los principales artífices de traer a Pamplona a Remacha. Finalmente "Don Félix" es el empresario Félix Huarte que ejerció además como Vicepresidente de la Diputación Foral de Navarra durante los años 1964 a 1971. 
Respecto al cachet, hemos establecido para esta primera tournée por España, uno moderado de 15000 pts. para la orquesta y el solista, si lo hubiera, que podrían elegir entre el violinista español Gerardo Gómez Casais, el violista Jean Rieber y la violoncellista francesa Genevieve Teulieres.

En cuanto a programas, adjunta encontrará una hoja con lo que les proponemos, así como las obras de solistas.

Dada la fecha en que estamos, nos interesaría conocer con la mayor urgencia lo que al respecto puedan decidir en uno u otro sentido. Por consiguiente, le agradecería que me hiciese saber dicha decisión lo antes posible.

Quedo entretanto, de Vd. affmo.

A. Alfonso

\section{Carta de Fernando Remacha al Rvdo. Dom Bergés}

Pamplona, 21 de Febrero de 1959

Rvdo. Dom Bergés

Leyre

Mi distinguido amigo: le agradezco mucho sus gestiones que tan buen resultado han dado ya que podemos contar con Dom Gajard y Potiron en las fechas previstas.

Respecto al contenido de las cinco lecciones, mejor que conferencias, que pueden organizarse, creo que dada la práctica que esos dos Sres tienen se les puede dejar en completa libertad para que elijan los temas a desarrollar. Ahora bien: a Dom Gajard se le puede indicar que convendría preparar un a Misa para el día 8 que se podría cantar en Leyre por los cursillistas ya Mr. Potiron que se pretende que tengan nociones sólidas del acompañamiento del Gregoriano, cosa un poco pretenciosa tratándose de un curso de 5 días. Creo que es lo único que les podemos indicar ya que ellos van a ser los Maestros y nosotros los discípulos y es a ellos a quien hay que pedir consejo.

De todas formas para la propaganda del Curso hay que dejar los temas bien determinados para que el que quiera venir sepa a lo que viene.

Haré las gestiones para traer a Dom Pujols si es que ha regresado de África para entonces. Esto lo tramitaré ahora mismo.

Espero que me envíen Vds. algún motivo alegórico del Monasterio para incluirlo en los carteles de propaganda, cosa que le propuse al P. Ramos.

Con el mayor respeto le saluda afectuosamente su afmo. amigo ${ }^{36}$.

\section{Carta de Martín Lipúzcoa a Fernando Remacha}

Madrid, 25 de marzo de 1959

\section{Sr. D. Fernando Remacha}

Tudela

Muy estimado Sr. Director: a mi hermano le dicen en Madrid que presente Vd. solicitud dirigida al Sr. Ministro de Educación para que conceda la cantidad proyectada para la Semana Gregoriana.

En el Palacio Arzobispal recomiendan, por ser más eficaz, $1^{\circ}$ publicar el anuncio de la Semana en el Boletín Oficial diocesano del próximo abril junto con las letras comendaticias del Sr. Arzobispo (?)

De esto se encarga ya el Sr. Secretario de Cámara.

$2^{\circ}$ Cuando esté ya publicado el programa del Cursillo, enviar este junto con una carta del Sr. Arzobispo a todos los Boletines

Diocesanos de España y cuantas revistas y publicaciones eclesiásticas interesen.

La carta me la facilitará el dicho Secretario y su copia con los programas los enviaremos nosotros.

Creen que esta es la forma más eficaz y quizá la única para que no falle el anuncio y a la vez se consiga gratuitamente.

Y deseándole unos días placenteros, con un saludo a su distinguida esposa e hijos, se despide afectuosamente su buen amigo Martín Lipúzcoa

36 Tal y como señala Flamarique: "El P. Fermín Ramos O.S.B. procedente de Silos y residente en ese momento en Leyre dio en Pamplona una Conferencia-Concierto el 25.06.1959 en el Salón de Conferencias del Museo de Navarra: FLAMARIQUE, 2011: 32 . Dicha conferencia mereció atención en la prensa local durante varios días $(21,24,25$ y 26 de junio) donde no se escatimaron elogios sobre el ponente, al que se calificaba de "joven y culto monje organista","erudito conferenciante", "especialista en canto gregoriano"... 


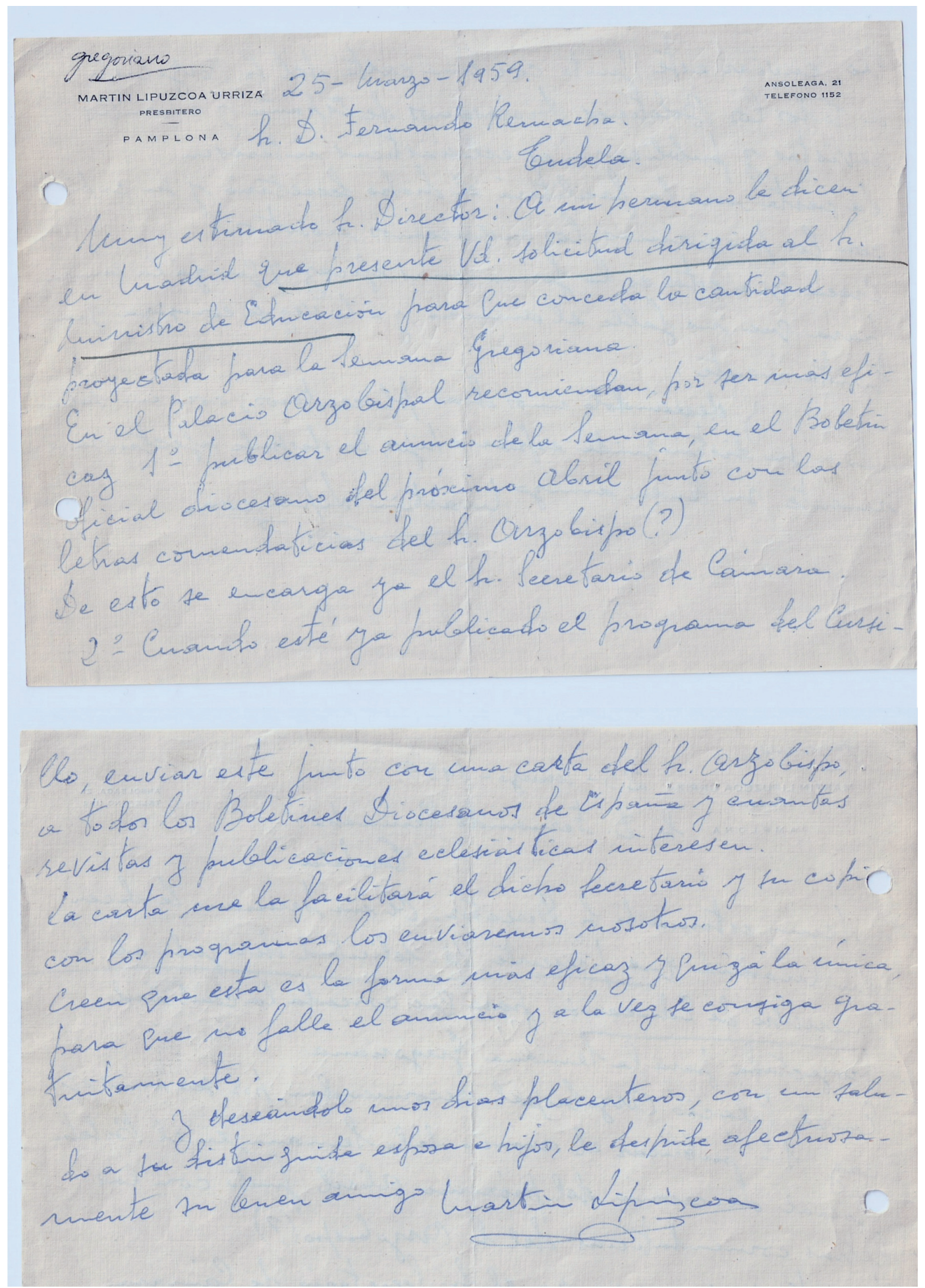

Figura 2: manuscrito de la carta de Martín Lipúzcoa a Fernando Remacha (25.03.1959) 


\section{Carta de Fernando Remacha al Rvdo. Dom Bergés}

Pamplona, 30 de Abril de 1959

Rdo. Padre Dom Bergés

Leyre

Rdo. Padre y estimado amigo: ya tengo el dinero para pagarle su suscripción a la Revue Gregorienne (130 Ptas.).

Le incluyo una hoja del Journal Musical Francais para que trate Vd de que anuncien el programa de la Semana Gregoriana (que le ruego traduzca Vd al francés y en tamaño que marco en la última página con lápiz azul. También le envío la dirección de la Revue Musicale con el mismo objeto y encargando otro anuncio del mismo tamaño. Ambos anuncios convendría que saliesen en su número de Junio. Más adelante no publican otros hasta Octubre. Todos los gastos en francos puedo pagárselos inmediatamente, pues los de la Cátedra Huarte es un fondo aparte y dispongo de dinero en todo momento.

Perdone que le moleste tanto pero no se puede conseguir anunciar en Francia sino por medio de presonas que estén dentro del país.

Muchas gracias por todo y le saluda afectuosamente su buen amigo.

\section{Carta de Fernando Remacha a Jesús Guridi}

Pamplona, 17 de Marzo de 1959

Ilmo. Sr. Dn Jesús Guridi

Director del Real Conservatorio de Música de Madrid

Mi distinguido amigo: por encargo del Patronato que ahora se ha hecho cargo de organizar conciertos con la Orquesta de Santa Cecilia, me dirijo a Vd. para rogarle que nos preste el archivo del Conservatorio, como ya lo hizo otras veces, el material de Orquesta de la Sinfonía del Nuevo Mundo de Dvorak.

Esperando nos pueda Vd. hacer ese favor y con gracias anticipadas que de Vd. atto. 


\section{Carta de Federico Sopeña a Fernando Remacha ${ }^{37}$}

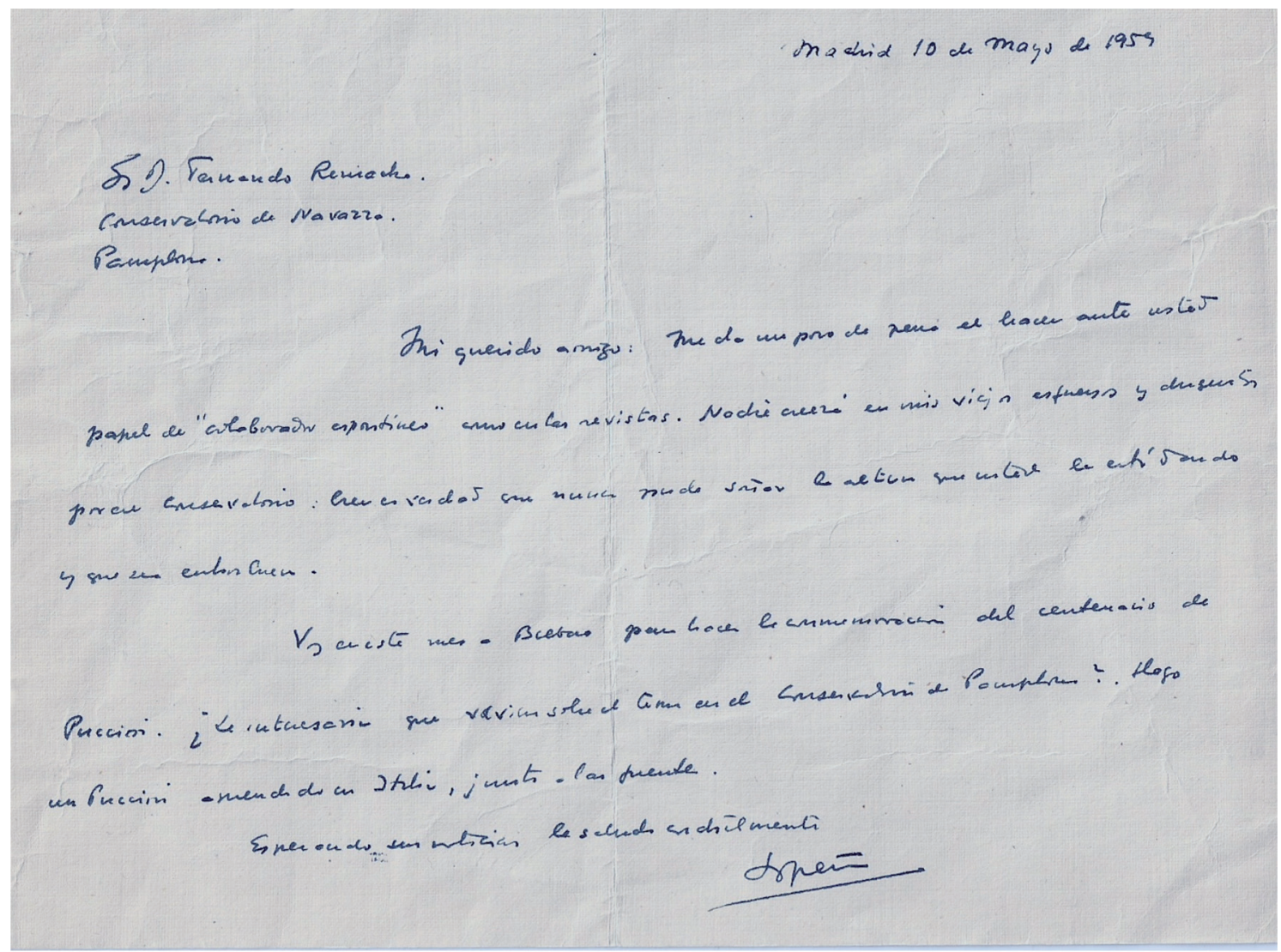

Figura 3: carta de Federico Sopeña a Fernando Remacha (10.05.1959)

\section{Carta de Fernando Remacha a Federico Sopeña}

Sr. Dn Federico Sopeña

Pamplona, 15 de mayo de 1959

Madrid

Mi querido amigo: le agradezco sus palabras de aliento acerca de la marcha de este Conservatorio y desearía que algún día pudiéramos charlar sobre ese tema.

Su proposición de dar la conferencia sobre Puccini no es posible aceptarla por ahora ya que este mes con el $8^{\circ}$ concierto del ciclo quedan agotadas para el año que viene, y en la "Cátedra Huarte", donde tengo completa libertad de acción prepare Vd. algo para 6 lecciones y que se refiera a la Música religiosa española. Creo que será un tema que pueda interesarle y celebraría que Vd. lo aceptase.

Espero sus noticias y hasta entonces le saluda afectuosamente.

37 En el caso de esta carta, al haber partes de una extrema dificultad para comprender la letra de Sopeña, se opta por poner directamente el manuscrito. 


\title{
Carta del Centro de Cultura por Correspondencia a Fernando Remacha
}

San Sebastián, 30 de mayo de 1959

\author{
Sr. D. Fernando Remacha \\ Director del Conservatorio de Música \\ "Pablo Sarasate" \\ Pamplona
}

Estimado señor:

En nuestro poder su atenta de fecha 11 del actual.

Nos agrada sobremanera su amable ofrecimiento de un curso de ARMONIA del que es autor, lamentando no poder aceptarlo por disponer ya de un original preparado para la imprenta.

Reiterándole nuestras gracias, quedamos de usted muy atentamente

CENTRO DE CULTURA POR CORRESPONDENCIA CCC

\section{Carta de Agustín González Acilu a Fernando Remacha}

Madrid, 14/6/67

Querido maestro:

Como le anunciaba en mi última del día 29 del mes pasado, le envío una fotocopia de mi obra junto a otro ejemplar para el Sr. Troncoso. Por ella verá Vd. la realización conseguida con material fonético, existente éste en el texto. Me gustaría explicarle personalmente la obra pero ante esta imposibilidad le daré algunos datos acerca de ella. Estos son los siguientes:

$1^{\text {a }}$ PARTE del 1 al 5. a) el texto se lee en la altura más cómoda, observando fielmente las intensidades $(\ldots)^{38}$, con su correspondiente vibración de forma articulada (no sonora). b) Proporcionar los espacios fónicos (I, II, III)

c) Observar las cadencias y articulaciones fónicas (...)

d) Observar los grupos fónicos más importantes (...) en cuanto al ritmo del texto.

e) Acentuación rítmica de la estructura de cada grupo fónico (...)

La función de la primera parte consiste en que el auditorio conozca el valor semántico del texto.

$2^{\text {a }}$ PARTE. Del 6 al 50. La tesitura (dentro de la mayor comodidad y de forma articulada) se divide en cuatro alturas (...), alturas irregulares entre sí, es decir, "no fijas o temperadas". Estas alturas derivan exclusivamente de su correspondiente dinámica. Las sílabas (siempre articuladas) se ejecutan en "mode tendî" (base tensa) hasta "mode croissant" (base creciente) base de la articulación de la lengua francesa.

$3^{\text {a }}$ PARTE. Del 51 al 68 recoge la ordenación por interpolación de relativas duraciones y acentuaciones de cada grupo o fragmentos fónicos (...) representados de la forma siguiente (...).

Del 74 al 89 recoge los principales grupos rítmicos del texto (...) intercalados entre sí.

$4^{\text {a }}$ PARTE. Del 91 al 111 recoge el compendio de (...), es decir los elementos más importantes del contenido musical fonético del texto en seis órdenes (...). La duración depende de su altura. El material sonoro-instrumental está elegido de una control de división de las 16 cuerdas (en glissandi), control relacionado con la altura derivada de la dinámica del texto.

Del 112 al 127 está compuesto de sílabas que corresponden a tres órdenes. Acústica, perceptibilidad y Frecuencia. El 128, 129 y 130 constan de estos mismos órdenes, pero en su núcleo mínimo, es decir el fonema.

De la organización instrumental diré que el aplicar los cuartos de tono (por afinación alterada de dos de los instrumentos) obedece a la necesidad de disturbar el principio físico armónico destruyendo de esta forma una interválica que no obedece ni corresponde a la interválica fonética.

El material sonoro instrumental procede de la división de los semitonos, por lo que he trabajado con 24 sonidos con un criterio controlado de los mismos.

38 Se omiten algunas grafías que dibuja González Acilu y que pueden verse en la imagen escaneada. 
La articulación de los instrumentos está en función de la base de articulación de la lengua francesa. Los espacios instrumentales corresponden a la separación de grupos fónicos.

La obra la estamos ensayando para su próxima grabación en la Radio. La voz hace José Luís Ochoa de Olza y el resultado sonoro va siendo el previsto.

Asimismo le diré que dicha obra está sorprendiendo a cuantos explico y enseño la partitura. Por otra parte, ante la experiencia de esta primera obra se abre ante mí unas posibilidades muy amplias en cuanto al binomio sonido-texto. Posibilidades que como puede observarse derivan de un criterio físico del texto.

Entre mis proyectos de nuevas obras hay uno que desearía realizar rápidamente. El proyecto es el siguiente:

Voces (coro) solo y orquesta.

Texto: Explicación por el "solo" del castellano (fonética y fonologicamente) a anglo-hablantes, franco-hablantes y germano-hablantes.

Forma: El "solo" enseña la formación de nuestra lengua con signos de la I.PH.A (Internacional Fonética Asociación), comenzando por los núcleos simples, es decir, fonemas, sílabas, palabras...modificaciones entre sí (fono sintaxis) llegando al terreno de la Semántica que podría consistir en una lección de fonética y fonología de la lengua castellana. La tensión de la obra me daría la progresiva perceptibilidad de la (en un principio) confusión sonoro-fonética de las tres lenguas.

La articulación del grupo instrumental irá paralela a la que corresponde a cada una de las cuatro lenguas: francés, inglés, alemán y español.

Como le digo más arriba me gustaría explicarle personalmente todo esto con más detalles.

Por todo lo expuesto ¿cree Vd. que hay este año la posibilidad de obtener un encargo de la Diputación u otro organismo? En este curso 1966-67 termino la beca de Preparación a Cátedra que consistía en 3000 pesetas al mes.

Si usted viese alguna posibilidad, le agradecería me lo comunicase.

Afectuosamente le saluda. 
$\therefore \quad$ Madind $14 / 6 / 67$

Querido maetro:

SRyor Fernando Remacha

Como le anunciaba, en mi ultima del dia 29 del mes hasado, le envío una fotoeopia de mi obra junto a otro ejemplar para el f? Troneoso. Pr ella verá Vd, la realiza.

cion cousegrida en material fonético; exisfente ète en el texto. Me gustaria explicarle pertonalmente la oba prero ante esta imposifilidad le daré alguno datos acerea de ella. Esto Son los siguientes:

1- PARTE del (1) al (5). a) el texto fe lee en la altura mas cómoda, observando fielmente las intensidades, $(0, \infty, 0)$ con on corresfondiente vibracion de forma artienlada (Mo fonorizada). b) proporeionar los espacios fonies (I. II. III) en enanto al zitmo del texto.

e) acentuacion ritmiea de la estuctura de cada quipo fonieo La funcion de la 1 a frarte contiste en que el anditorio conozea el balor Semantieo del texto.

2a PARTE Del (6) al (50). La texitura (dentro de la mays como didad o de forma artienlada) ie divide en enatro alturas, $($ alturas irregulares entre si, es deeir, "no fijas o temprezadas". Estas alturas derivan exelustivamente de "Su" conespondiente" dinámiea. Las silabas (fiemfre artienladas) te ejeentan en "mode tendu" (bare tenta) hasta "mode eroissant" (bare ereciente) bare de artienlacion de la lengua paneesa. 
3a-PARTE del (51) al (68) recoge la ordenacion for interpolacion de relativas duraciones is acentuaciones de rada grupso 0 pagmentos fonieos $(>, \gg, \gg)$ ) refretentados de la forma signiente: $\left.\gg, \vec{p}_{1},\right\rangle_{1}$.

Del (74) al (89) reeoge los finineipales grupos ritmies del texto $(\Longrightarrow, \equiv)$ interealados entre si.

4IPARTE Del (91) al (111) reeoge el comfrendio de $>,>, \gg$. $\circ$, K. OF, $=$, es deeir, los elementos mas imprortantes del contenido musical fonetico del texto en seis ordewes La duracion depende de fu altura. El material sonoro-instumental, está cleqido de un control de division de las 16 enerdas (pu glissandi), control relacionado fon la altura; derivada de la dinamiea del texto. Lel (112) al (127) está compresto de silatas que couresponden a

tres odenes. Acustiea, Pereeptifilidad \& Freenencia. El 128 (129) I (130) constan de éstor mismos ordenes, pero en in inieleo minimo, es decir, el fonema.

We la ogganizaeion instrumental diré que el afliear los enartos de tono (por afinacion alterada de dos de lo instru mentos) obedece a la mecesidad de disturbar el firicieifio fisieoarmonieo destrugendo de ista forma una intervaliea que no obedece mi corresponde a la interbaliea forietica. El material sonoro instrumentale frocede de le division de los temitons: fur lo que he trabajado con 24 sonidos con un eriterio controlado de los mismos.

La artienlacion de los instumentos sità en funcion de la base de artienlacion de la lengua pancesa. Los espacios instumentales correstronden a la separación de gumpos forícos 
La obre la estanos ensayando para su froxima grabeion en la Radio. La voz hace Lose tuis Oehoa de Olza y el

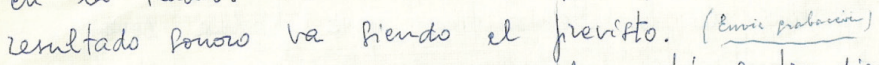

trimismo le diré que dicha obra está forfrendiendo a enountos explies y enseño la partitura. Por otre parte aute la experiencia de esta frimera oba fe abre ante mi unas frosibilidades puny amflias en enanto al binomio sonido-texto. Positilidades que conno mede obtervarse derivan de un eriterio fisieo del texto.

Eutre mis frogeetos de muevas ohas hay uno que derearia realizar rapidamente. El progeeto as el figniente:

Voces (ero) Solo j Orqueta.

Texto: Explicacion for el "Solo" del castellano (fonctica g fonologieamente) a auglo-hablantes, pareo-hablantes, I fermano-hablantes. Forma El solo" enteña la fromunciacion de unetra lengua con signos de la I.PH.A (Internacional, Fontica, Arociacion)

comenzando for los pueless simples; as decir, fonemas, silabas, palabes, sizremas..... modifieandols entre si (foriosintaxis) llegando al terreno de la Semantica que podia consistir en una leceion de fonitica of fonologia de la lengua castellar. La tension de la oba. me daria la fropresiva pereeptitilidad de la (en un friveipio) "confusion" sonoro-forietica de las tres lenguas. corveponde a pada una de las puatro lenguas: Hances, Ingles Aleman Jouno le digo mas arriba me qustaria eypliearle personalmente todo esto con mas detalles.

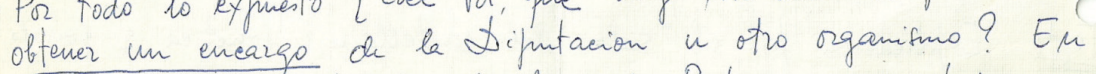
este enzro 1966-67 termino la beea de Preparacion a petecha que consistia en 3000 hts al pues.

Si Vd viere alquma posibilidad, le agradiceria me lo comminerse Afectrosamente le salnde frustur fortas

Figura 4: Carta de Agustín González Acilu a Fernando Remacha, 14.06.1967 


\section{Bibliografía}

Andrés, Marcos, Vida y obra de Fernando Remacha. Tesis doctoral, Universidad de Valladolid, 1997.

Andrés, Marcos, Fernando Remacha. El compositor y su obra. Madrid, ICCMU, 1998a, 213.

Andrés, Marcos, "Selección de documentación en torno a Fernando Remacha", Revista del Centro de Estudios de Merindad de Tudela, 8 (1998b), 29-92.

Andrés, Marcos, "25 Años de la muerte de Fernando Remacha", Cuadernos de Música Iberoamericana, 18 (2009), 125-126, 128.

Andrés, Marcos, "El sistema compositivo de Joseba Torre (1968), análisis del proceso compositivo y análisis de la obra", Revista de Musicología, 33/1-2 (2010a).

Andrés, Marcos, "Cuatro cartas de Fernando Remacha (1898-1984) a Jorge Oteiza (1908-2003)", Príncipe de Viana, 71/249 (2010b), 75-85.

Andrés, Marcos, "Fernando Remacha Villar (1898-1984), In memoriam". En: Nueva creación musical en memoria de Fernando Remacha Villar. [Andrés, Marcos (coord.)]. Pamplona, Universidad Pública de Navarra, 2010c.

Arce, Julio, Música y radiodifusión. Los primeros años (1923-1936). Madrid, ICCMU, "Colección Música Hispana. Textos. Estudios”, 2008, 105.

Berrade, Jaime, La Orquesta Sinfónica de Navarra: aspectos socioeducativos en su devenir histórico. Tesis doctoral, Universidad Pública de Navarra, 2011.
Cureses, Marta, Agustín González Acilu. La estética de la tensión. Madrid, ICCMU, "colección Música Hispana. Textos. Biografías”, Edición 2a 2001.

Flamarique, Milagros, Semanas gregorianas de Pamplona: un proyecto en dos actos. Trabajo Fin de Máster, "Máster universitario en estudios avanzados en historia, espacio y patrimonio", Universidad Pública de Navarra, 05.09.2011.

Heine, Christiane, "Fernando Remacha (1898-1984), su despedida de la música", Revista de Musicología, 19/1 (1996), 240-241.

Palacios, María, La renovación musical en Madrid durante la dictadura de Primo de Rivera. El Grupo de los Ocho (1923-1931). Madrid, SEdeM, 2008, 114-118.

Remacha, Fernando, Curso de Armonía a distancia. San Sebastián, Poliglopone, 1964.

Sáinz de la Maza, Regino, "Sociedad de cursos y conferencias. Concierto de música contemporánea", Diario La Libertad (13.06.1933), 8.

Salazar, Adolfo, "Música moderna en la Residencia de Estudiantes", El Sol (14.06.1933), 5.

Sopeña, Federico, "La música en la ciudad: tradición y actualidad. El único conservatorio "funcional" de España", $A B C$ (15.10.1963), 20-23.

Temes, José Luis, Fernando Remacha. Obra sinfónica completa. Madrid, Verso-Comunidad de Madrid-Gobierno de Navarra, 2009.

Recibido: 28.11 .2012

Aceptado: 02.11.2015 